\title{
Lateral Lobe of the Prostate
}

National Cancer Institute

\section{Source}

National Cancer Institute. Lateral Lobe of the Prostate. NCI Thesaurus. Code C13092.

The prostate gland lobe that is located on the lateral side of the organ. 